$$
\begin{aligned}
& \text { CONF-970510-5 } \\
& \text { SANO97.0791C } \\
& \text { SAND- } 97-0791 C
\end{aligned}
$$

\title{
Stimulated Emission from Semiconductor Microcavities
}

\author{
Xudong Fan and Hailin Wang \\ Department of Physics, University of Oregon, Eugene, OR 97403 \\ H.Q. Hou and B.E. Hammons \\ Sandia National Laboratories, Albuquerque, NM 87185 \\ $541-346-4758$ (voice) \\ 541-346-4791 (fax) \\ RECEVIEO
APR 101997 \\ OSTI
}

Abstract

Studies of both emission and reflection spectra of a semiconductor microcavity near the lasing threshold provide important information on lasing mechanisms of semiconductor microcavities at low temperature and also reveal significant roles of exciton localization.

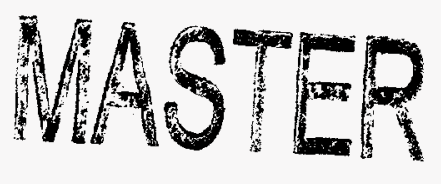




\section{DISCLAIMER}

This report was prepared as an account of work sponsored by an agency of the United States Government. Neither the United States Government nor any agency thereof, nor any of their employees, make any warranty, express or implied, or assumes any legal liability or responsibility for the accuracy, completeness, or usefulness of any information, apparatus, product, or process disclosed, or represents that its use would not infringe privately owned rights. Reference herein to any specific commercial product, process, or service by trade name, trademark, manufacturer, or otherwise does not necessarily constitute or imply its endorsement, recommendation, or favoring by the United States Government or any agency thereof. The views and opinions of authors expressed herein do not necessarily state or reflect those of the United States Government or any agency thereof. 


\title{
Stimulated Emission from Semiconductor Microcavities
}

\author{
Xudong Fan and Hailin Wang \\ Department of Physics, University of Oregon, Eugene, OR 97403 \\ H.Q. Hou and B.E. Hammons \\ Sandia National Laboratories, Albuquerque, NM 87185
}

Laser-like emissions from semiconductor microcavities at low temperature have attracted considerable attention recently because of the possibility of realizing a non-equilibrium condensate by using cavity-polaritons [1]. In this paper we present experimental studies of optical properties of a microcavity near the lasing threshold. We show that the minimum lasing threshold is achieved when the cavity is tuned significantly below the exciton line center. By comparing emission spectra with reflectivity spectra, we also show that well-resolved doublet in the emission spectra near the lasing threshold are not associated with cavity-polaritons. These results suggest that laser-like emissions from the microcavity are due to conventional stimulated emission processes with exciton localization playing a significant role.

The experiment is carried out in a microcavity consisting of 16 and $22 \lambda / 4$ stacks of $\mathrm{Al}_{0.11} \mathrm{Ga}_{0.89} \mathrm{As} / \mathrm{Al}$ As as the top and bottom mirrors, respectively. Four $130 \AA \mathrm{GaAs}$ quantum wells are placed at the center of a $\lambda$ cavity. Off-resonant pumping with $150 \mathrm{fs}$ pulses from a Ti:Sapphire laser is used for emission measurements. All measurements were performed at $10 \mathrm{~K}$.

Figure la shows reflection spectra of the sample when the cavity is at the exciton absorption line center. At the low excitation regime, the reflection spectrum is characterized by two well-resolved cavity-polariton resonances. With increasing exciton densities, the normal mode splitting decreases due to exciton absorption saturation. At the high excitation regime, the splitting collapses and the reflectivity spectrum is characterized by the empty cavity resonance as shown by the dashed curve in Fig. 1a.

Figure $1 \mathrm{~b}$ shows corresponding emission spectra near the lasing threshold. Two wellresolved resonances are observed. A distinct threshold behavior is also observed for the resonance at the higher energy as shown in Fig. 2. The frequency of this resonance, however, is 
Fan et al., Stimulated emission...

at the position of the empty cavity resonance instead of the position of the cavity-polariton. Similar behaviors have also been observed when the cavity is tuned away from the exciton line center with the laser-like emission always at or near the frequency of the empty cavity resonance.

It is important to point out that the two emission resonances shown in Fig. $1 \mathrm{~b}$ are not associated with cavity-polariton resonances in reflection spectra since reflection spectra at densities near or above the lasing threshold should be characterized by the empty cavity resonance shown in Fig. 1a. The appearance of two resonances in the emission spectra can be understood by using a linear dispersion model that includes effects of exciton localization. In this model, one resonance in the emission spectra is associated with the empty cavity resonance and will go above the threshold with increasing intensities. The other resonance is associated with emission resonance in the absence of the cavity. This resonance is Stokes-shifted to energies below the exciton line center due to exciton localization (a simple estimate gives an exciton Stokes shift of 4 $\mathrm{meV}$ ). The actual energy position of this resonance will also be influenced by mode pulling of the cavity.

Effects of exciton localization is also evidenced in the dependence of the threshold intensity on detunings between the exciton and cavity (see Fig. 3). The minimum threshold is achieved when the cavity is tuned to $4 \mathrm{meV}$ below the exciton absorption line center. The corresponding minimum threshold intensity $\left(50 \mathrm{~W} / \mathrm{cm}^{2}\right)$ corresponds to an estimated exciton density in the range of $10^{10} / \mathrm{cm}^{2}$, which is expected to be below the exciton Mott density. At much higher densities, stimulated emissions are expected to be due to electron-hole plasmas. Transitions between these two different density regimes will also be presented.

This work is supported by AFOSR.

Sandia is a multiprogram laboratory operated by Sandia Corporation, a Lockheed Martin Company, for the United States Department of Energy under Contract DE-ACO4-94AL85000. 


\section{Fan et al., Stimulated emission...}

Figure Captions:

Fig. 1 (a) Reflection spectra at the low (solid line) and high (dashed line) excitation regimes. (b) Emission spectra near the lasing threshold. Dot-dashed, dashed, and solid spectra are obtained with increasing input intensities.

Fig. 2 Spectrally-integrated intensities of the two emission resonances shown in Fig. $1 \mathrm{~b}$ as a function of the input intensity. The circles and triangles are for resonances at the higher and lower energies, respectively.

Fig. 3 Lasing threshold behavior at various detunings between the exciton line center and the cavity. $\delta=\lambda_{c}-\lambda_{x}$ is the wavelength difference between the cavity resonance and the exciton line center. 
Fan et al., Stimulated emission...

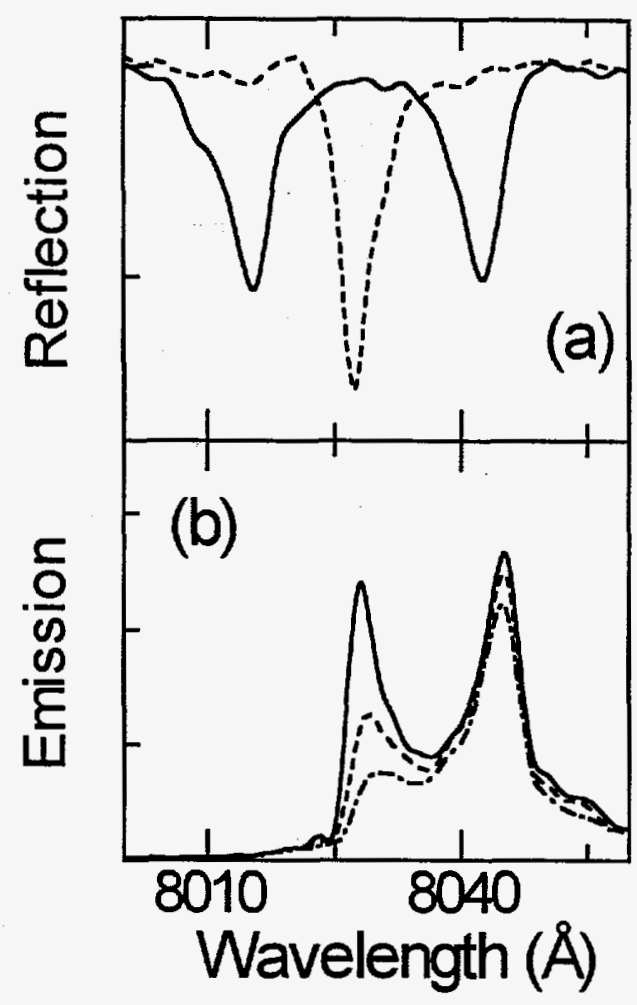

Fig. 1 
Fan et al., Stimulated emission...

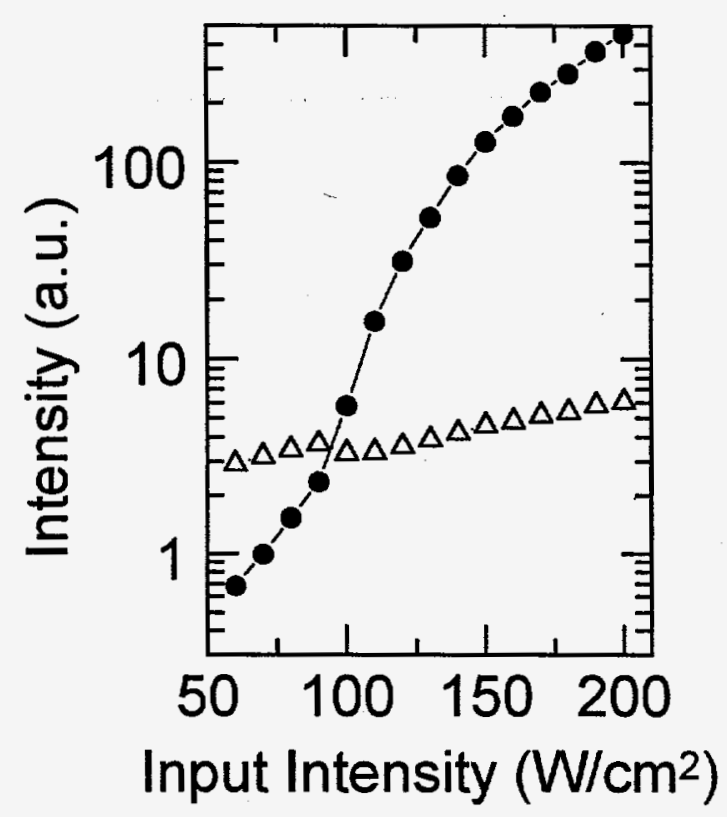

Fig. 2 


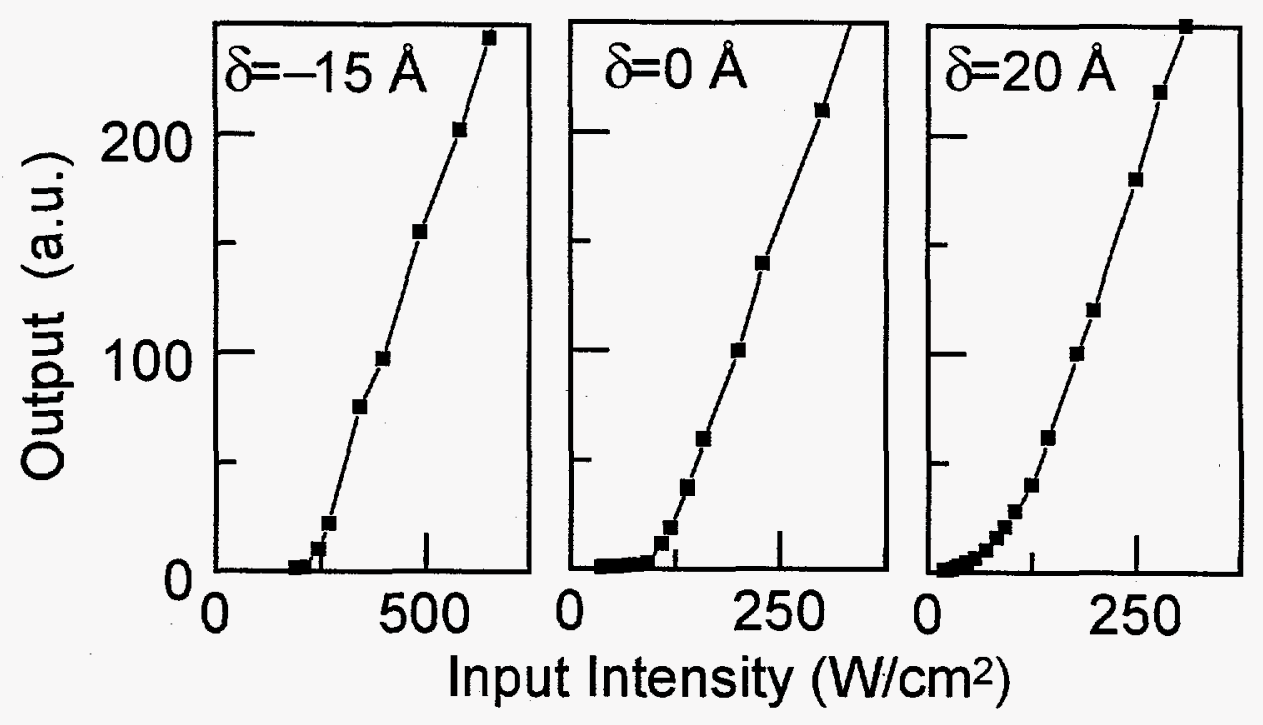

Fig. 3 